\title{
Eritema nodoso: um relato de caso
}

Carina Manuela Machado Pereira*

\section{RESUMO}

Introdução: A gravidez, sendo um período de intensas alterações fisiológicas, induz frequentemente a alterações cutâneas cujo diagnóstico diferencial se revela desafiante, sobretudo quando se trata de patologias menos frequentes. Ao longo da gestação numerosas alterações cutâneas podem ocorrer: as consideradas fisiológicas, as dermatoses específicas da gravidez e as que representam agravamento ou melhoria de dermatoses pré-existentes.

Descrição de caso clínico: Mulher de 26 anos, grávida, G3P2, vigiada numa Unidade de Saúde Familiar, com antecedentes pessoais de asma desde a infância, controlada no momento, sem antecedentes familiares de relevo. $\mathrm{Na} 11^{\text {a }}$ semana de gestação recorreu à consulta não programada por aparecimento de lesão eritematosa no membro inferior, com aumento progressivo de tamanho, dolorosa ao toque, não pruriginosa, com rubor e edema associado e uma semana de evolução. Colocada inicialmente a hipótese de tromboflebite foi encaminhada para o serviço de urgência hospitalar, tendo sido realizado o diagnóstico de eritema nodoso na gravidez.

Comentário: O caso clínico descrito pela autora salienta as alterações cutâneas que surgem frequentemente no contexto de consultas de saúde materna nos cuidados de saúde primários, chamando a atenção para uma alteração menos comum: o eritema nodoso. Esta patologia é uma alteração cutânea com vários fatores etiológicos possíveis, cujo reconhecimento orienta o médico, tranquiliza a gestante e permite estabelecer condutas adequadas, dado o caráter benigno e autolimitado deste quadro clínico.

Palavras-chave: Gravidez; Eritema nodoso.

\section{INTRODUÇÃO}

A gravidez é um período de intensas alterações fisiológicas que conduzem frequentemente a alterações em diversas funções e órgãos. As alterações cutâneas são algumas das mudanças que podem ocorrer com a gravidez, podendo ser divididas em alterações fisiológicas gestacionais, dermatoses específicas da gravidez e dermatoses modificadas pela gestação. ${ }^{1-2}$ As primeiras são as mais comuns, daí serem as primeiras a serem colocadas como hipótese de diagnóstico aquando do aparecimento de gestantes com alterações cutâneas, no âmbito das consultas de medicina geral e familiar. Em segundo lugar aparecem as dermatoses específicas da gravidez, às quais deve ser dada particular atenção dado o potencial que têm de comprometer o bem-estar materno e/ou fetal. Menos frequentes são as dermatoses pré-existentes, suscetíveis de agravamento ou melhoria durante a gravi-

*USF Oceanos, ULS Matosinhos. dez, como é o caso do eritema nodoso (EN)..$^{2-3}$

As doenças dermatológicas relacionadas com o período gestacional podem estar restritas à pele e mucosas ou expressar um comprometimento sistémico, pelo que a gestão dessas situações requer uma interligação multidisciplinar entre obstetras, dermatologistas e médicos de medicina geral e familiar.

A exposição deste caso clínico torna-se pertinente, dada a dificuldade de diagnóstico diferencial que as alterações cutâneas impõem e pela menor frequência de casos de EN em comparação com as restantes alterações cutâneas associadas à gravidez.

\section{DESCRIÇÃO DO CASO \\ Identificação}

Mulher grávida, caucasiana, com 26 anos de idade, com o $12^{\circ}$ ano de escolaridade e empregada de loja. O seu agregado familiar era constituído pelo marido e duas filhas, sendo uma família do tipo nuclear, da classe III de Graffar e estadio IV do Ciclo de Duvall. 


\section{Antecedentes pessoais}

Apresentava como antecedentes pessoais asma diagnosticada na infância, atualmente controlada. Como antecedentes cirúrgicos, de referir a colocação de parafuso epifisário de fixação no fémur pós fratura, em 2002, e uma amigdalectomia, em 2001, sem intercorrências.

Quanto à história obstétrica: G3P2, dois partos eutócicos de termo em 2007 e 2010. As gestações decorreram sem intercorrências, nomeadamente, sem qualquer ocorrência de episódios de EN.

Tratava-se de uma utente que não praticava atividade física, fumadora até ao início da gestação atual (carga tabágica 20 UMA), com cessação tabágica também durante as gestações prévias, sem hábitos etílicos ou de consumo de drogas ilícitas.

Medicada com salbutamol (sistema pressurizado) em SOS, ácido fólico e iodo.

Sem antecedentes familiares de relevo.

\section{História da doença atual}

\section{5 de fevereiro de 2016}

\section{Consulta não programada na USF}

Grávida na $11^{\mathrm{a}}$ semana de gestação, que recorreu a consulta pelo aparecimento de tumefação na região tibial anterior da perna direita, com rubor, edema e dor associados, com cerca de uma semana de evolução. Foi colocada a hipótese de tromboflebite, tendo sido a doente encaminhada para o serviço de urgência de obstetrícia do hospital de referência.

\section{Serviço de urgência do hospital de referência}

Foi observada pelas especialidades de obstetrícia e medicina interna, tendo a doente nesta altura feito referência, além da tumefação na perna direita, a uma outra no membro inferior esquerdo com aparecimento no dia anterior. Descreveu as tumefações como sendo dolorosas ao toque, não pruriginosas e com um aumento progressivo de tamanho.

A doente negou febre, perda ponderal ou queixas articulares adicionais. Sem dor abdominal, alterações do trânsito gastrointestinal ou geniturinárias. Sem queixas de xerostomia/xeroftalmia. Sem episódios sugestivos de síndroma de Raynaud.

Negou, ainda, toma de medicação de novo, contacto com pessoas doentes e/ ou viagens recentes. Sem his- tória familiar de doenças autoimunes.

Referiu uma história de infeção das vias respiratórias superiores, com necessidade de ida ao Serviço de Atendimento a Situações Urgentes, da área de residência, a $02 / 01 / 2016$, onde foi medicada com amoxicilina, ibuprofeno e paracetamol.

Ao exame objetivo apresentava-se hemodinamicamente estável. Nos membros inferiores observaram-se duas lesões eritematosas nodulares com elevação: uma na região tibial anterior da perna direita, com cerca de $3 \mathrm{~cm}$ de maior diâmetro e outra na região medial da perna esquerda, com cerca de $1,5 \mathrm{~cm}$. Sem edemas periféricos (mas com edema associado) (Figura 1).

Analiticamente apresentava: hemoglobina 12,2g/dl; leucócitos $6,79 \times 10^{9} / \mathrm{L}$; plaquetas $187.000 / \mathrm{mm}^{3}$; proteína $\mathrm{C}$ reativa $2,9 \mathrm{mg} / \mathrm{dL}$; função hepática e renal normais; urina II sem alterações.

Diagnosticado então EN na gravidez, tendo sido informada do curso benigno da patologia e dada indicação de realizar terapêutica analgésica com paracetamol, se necessário.

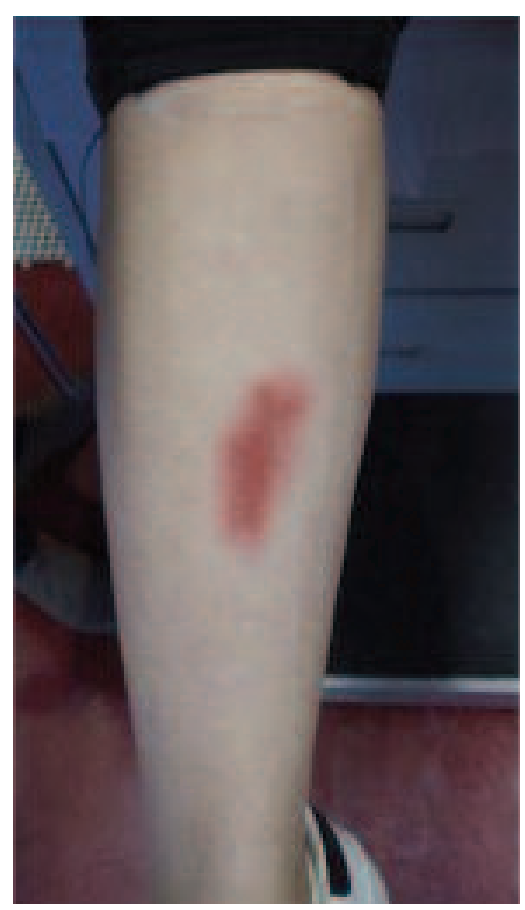

Figura 1. Lesão eritematosa nodular. 


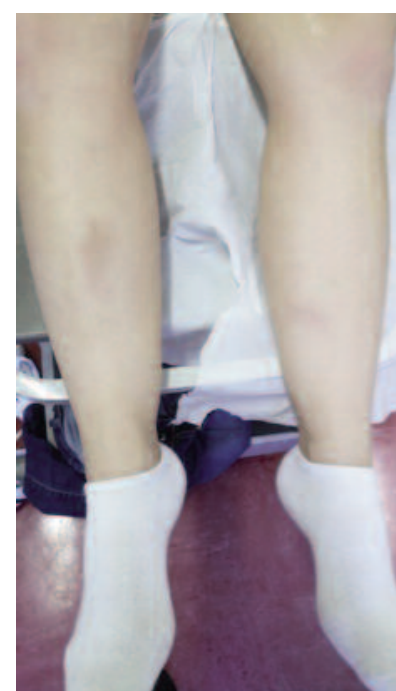

Figura 2. Eritema contusiforme.

\section{6 de abril de 2016}

\section{Consulta de saúde materna}

Gestante na $21^{\text {a }}$ semana de gestação, assintomática, apresentava ao exame objetivo hiperpigmentação nos locais atingidos previamente pelo EN - eritema contusiformis (Figuras 2 e 3).

Fez referência ao reaparecimento, duas semanas antes, de duas tumefações nos membros inferiores, com a mesma localização e características do episódio de fevereiro, nomeadamente dor e rubor que melhoraram progressivamente, sem instituição de qualquer terapêutica. Associou o agravamento das lesões à toma de amoxicilina e desloratadina prescritos em contexto hospitalar, à 15a semana de gestação, por novo episódio de infeção respiratória.

Posteriormente, com a evolução do quadro verificou-se a manutenção de eritema contusiformis, sem outros sintomas ou sinais associados. A restante gestação decorreu sem outras intercorrências; às 39 semanas de gestação foi realizado um parto eutócico, tendo o recém-nascido apresentado ao nascimento peso e estatura dentro dos parâmetros considerados normais.

\section{COMENTÁRIO}

O diagnóstico das dermatoses que surgem durante a gravidez é um desafio, uma vez que muitas delas são ainda pouco entendidas e pela existência de sobreposição entre características clínicas e histopatológicas

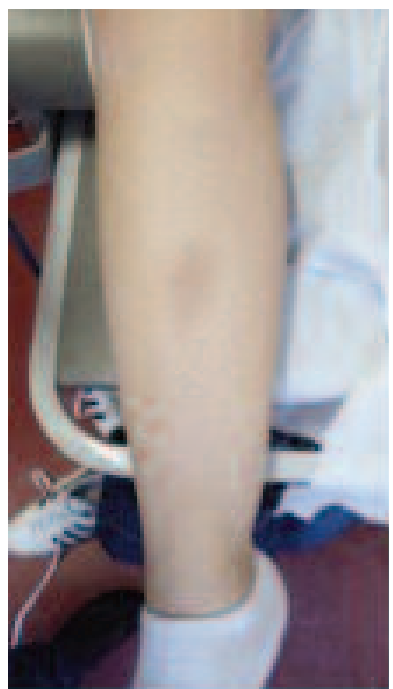

Figura 3. Eritema contusiforme.

das diferentes entidades. ${ }^{3-4}$

Embora raras, as alterações cutâneas específicas da gestação, como penfigoide gestacional e colestase intra-hepática da gestação, têm um elevado risco de efeitos adversos no feto e é importante distingui-las de doenças de base que se manifestam pela primeira vez durante a gestação ou que se agravam nesta fase, como é o caso do EN. ${ }^{2}$

O EN é uma das possíveis alterações cutâneas modificadas pela gestação, encontrando-se a sua prevalência neste período da vida entre os 4,6 e 6\%. Tende a ocorrer mais no primeiro trimestre de gestação. ${ }^{5} \mathrm{Em}$ bora possa ocorrer em qualquer idade, é mais comum na terceira e quarta décadas de vida. ${ }^{6}$

É um tipo de paniculite que afeta a gordura subcutânea e se caracteriza por um início súbito de nódulos eritematosos, arredondados, ligeiramente elevados, com 1 a $6 \mathrm{~cm}$ de diâmetro, dolorosos, não supurativos, classicamente na face extensora dos membros inferiores, mas podendo em alguns casos envolver tornozelos, coxas, braços, rosto, pescoço e tronco. Muitas vezes as lesões são associadas a sintomas sistémicos como febre, edema das pernas, artralgias e alterações gastrointestinais. A ulceração é incomum e resolve sem cicatrizes, mas pode deixar hiperpigmentação residual (eritema contusiformis)..$^{6-7}$

No caso clínico apresentado verificou-se o aparecimento de nódulos dolorosos na face anterior e medial 
dos membros inferiores, à semelhança do que é mais frequente acontecer. Inicialmente, aquando da ida à Unidade de Saúde Familiar a doente apenas fez referência e valorizou os nódulos de um membro pela sintomatologia mais exuberante e pela maior dimensão. Desta forma, foi levantada a hipótese de se tratar de uma situação clínica de tromboflebite. Posteriormente, após recolha de uma história clínica completa e de um exame físico global, foi observada a existência de um outro nódulo, no membro contralateral, com características similares, tornando menos provável a hipótese de uma causa vascular e levantando a hipótese diagnóstica de EN. Este aspeto chama a atenção para a importância da realização de uma acurada história clínica e um minucioso exame objetivo para realização de um correto enquadramento de sintomas/sinais por forma a ser possível o diagnóstico.

O EN é considerado o protótipo de "paniculite septal» pela inflamação dos septos no tecido adiposo subcutâneo; não está associado à vasculite, embora possa ocorrer inflamação e hemorragia em pequenos vasos. ${ }^{6,8}$

É associado a muitas condições subjacentes, mas a sua etiologia é desconhecida em metade dos casos (idiopático). As causas associadas a esta entidade clínica são apresentadas no Quadro 1.

A etiologia do EN durante a gestação não está, até ao momento, completamente compreendida, mas parece relacionar-se com mecanismos imunológicos em associação com alterações hormonais que ocorrem durante a mesma.

O EN é um padrão de reação cutânea inespecífico para uma variedade de antígenos, com inúmeros mecanismos imunomediados implicados. A maioria das evidências diretas e indiretas apoia o envolvimento de uma resposta retardada de hipersensibilidade de tipo IV a numerosos antígenos. ${ }^{8}$ Existem evidências para o papel dos complexos imunes no EN. A imunofluorescência direta das lesões mostra depósitos de imunoglobulinas e complemento nas paredes dos vasos afetados. Esse achado também pode explicar os sintomas associados de artralgia, febre e mal-estar. ${ }^{6}$

Alguns estudos sugerem ainda que o EN na gravidez se relaciona com uma reação de hipersensibilidade às diferentes hormonas (estrogénios e progesterona) que se elevam nesta fase. Quaisquer que sejam os mecanismos exatos, a gravidez parece disponibilizar um fun-
QUADRO I. Etiologias possíveis de eritema nodoso ${ }^{8}$

\section{COMUNS}

Idiopático (> 55\%)

Infeções: faringite estreptocócica (28 - 48\%), Yersinia spp.

(Europa), mycoplasma, chlamydia, histoplasmosis,

coccidioidomycosis, mycobacteria

Sarcoidose (11 - 25\%)

Fármacos: antibióticos (sulfonamidas, amoxicilina), contracetivos orais (3 - 10\%)

Gravidez (2 - 5\%)

Doença inflamatória intestinal: Doença de Crohn, colite ulcerosa $(1-4 \%)$

RARAS (< 1\%)

Infeções

- Virais: vírus herpes simples, vírus Epstein-Barr, vírus hepatite B e C, vírus da imunodeficiência humana

- Bacterianas: Campylobacter spp., rickettsiae, Salmonella spp., psittacosis, Bartonella spp., syphilis

- Parasitárias: amebíase, giardíase

Outras: linfomas, outras patologias malignas

do ideal para o desenvolvimento desta patologia, modulando o sistema imunológico.

Algumas gestantes apresentam um episódio de EN apenas na $2^{\text {a }}$ gestação, sem que o mesmo tenha ocorrido durante a primeira gravidez. Este aspeto pode indicar que outros fatores, além das hormonas sexuais femininas, possam desempenhar um papel no desenvolvimento do EN na gravidez, apresentando as hormonas um papel indireto na sua patogénese, possivelmente pela indução de um ambiente que é favorável ao seu desenvolvimento. ${ }^{7}$

Assim, no acompanhamento deste quadro clínico foi interessante verificar como, analisando de forma mais detalhada a história clínica da gestante, se encontraram possíveis fatores contribuintes para o aparecimento desta alteração cutânea: não só a gravidez em si, mas também o desenvolvimento inicial do quadro de EN após uma infeção das vias respiratórias superiores medicada com amoxicilina e desloratadina; também o reaparecimento cerca de um mês depois, das alterações cutâneas com as mesmas características, 


\section{QUADRO II. Diagnóstico diferencial das alterações cutâneas na gravidez²}

Alterações cutâneas fisiológicas da gravidez

Pigmentação - hiperpigmentação (difusa com acentuação nas aréolas e linha nigra)

\section{Melasma}

Tecido conectivo - Estrias

Sistema vascular - Edema, varicosidade/hemorróida, aranhas vasculares, telangiectasias, eritema palmar, hiperemia e hiperplasia gengival, granuloma piogénico

Função glandular - Aumento da função da glândulas écrinas (exceto palmas), diminuição da função de glândulas ápocrinas, distúrbio da função das glândulas sebáceas (acne gravídica e glândulas de Montgomery)

Cabelos - Hipertricose, eflúvio telógeno pós-parto, alopecia androgenética pós-parto

Unhas - Aumento da fragilidade, onicólise, hiperceratose subungueal, estriações

Doenças cutâneas específicas da gravidez

Penfigoide gestacional (sinónimos: pápulas e placas urticadas pruriginosas gestacional, eritema tóxico gestacional, rash toxémico gestacional)

Erupção polimórfica gestacional

Erupção atópica gestacional (sinónimos: prurigo gestacional, foliculite pruriginosa gestacional, eczema gestacional)

Colestase Intra-hepática gestacional (sinónimos: colestase obstétrica, prurido gravidarum)

Doenças cutâneas modificadas pela gravidez

\begin{tabular}{|c|c|}
\hline \multirow[t]{7}{*}{ Infeções } & Candidoses \\
\hline & Herpes simples \\
\hline & Varicela \\
\hline & Herpes Zoster \\
\hline & Tricomoníase \\
\hline & Condiloma acuminado \\
\hline & Hanseníase \\
\hline \multirow[t]{4}{*}{ Autoimunes } & Lúpus cutâneo crónico \\
\hline & Lúpus eritematoso sistémico \\
\hline & Esclerose sistémica \\
\hline & Dermatomiosite/polimiosite \\
\hline \multirow[t]{2}{*}{ Tecido conjuntivo } & Ehlers-Danlos \\
\hline & Pseudoxantoma elástico \\
\hline \multirow[t]{5}{*}{ Tumores } & Granuloma piogénico \\
\hline & Dermatofibromas, leiomiomas, queloides \\
\hline & Nevos \\
\hline & Melanoma \\
\hline & Micose fungoide \\
\hline \multirow[t]{4}{*}{ Miscelânea } & Dermatite atópica, acne e psoríase \\
\hline & Eczema dos mamilos e dermatite de contacto \\
\hline & Eritema multiforme, eritema nodoso e acantose nigricans \\
\hline & Porfíria cutânea tardia \\
\hline
\end{tabular}


após nova infeção respiratória e novo curso de antibioterapia com amoxicilina.

Geralmente os sintomas tornam-se mais intensos em uma a duas semanas e, em seguida, resolvem-se espontaneamente em uma a seis semanas, podendo em alguns casos demorar cerca de 12 semanas para resolver completamente. ${ }^{6-7}$

O diagnóstico diferencial com outras alterações cutâneas que ocorrem na gravidez, nomeadamente com as alterações fisiológicas gestacionais, com as dermatoses específicas da gravidez e com outras dermatoses alteradas na gestação, como as apresentadas no Quadro 2, é fundamental para um diagnóstico precoce e para uma abordagem terapêutica dirigida.

A abordagem mais comum é o tratamento de quaisquer distúrbios subjacentes e a terapia de suporte. A doença geralmente tem um curso benigno, autolimitado, em que meios não farmacológicos, como o repouso e o uso de meias de compressão elástica, habitualmente são suficientes para controlo sintomático. Se estas opções não são suficientes para aliviar os sintomas, outros regimes medicamentosos podem ser considerados. Os anti-inflamatórios não esteroides podem ser utilizados em certas situações, embora os obstetras tendam a evitar o uso de aspirina. Os corticosteroides sistémicos também podem ser usados durante o segundo e terceiro trimestres da gravidez. ${ }^{6}$

Ao escolher um tratamento farmacológico numa doente grávida é importante pesar os riscos e benefícios em cada caso, a fim de encontrar a opção de tratamento mais adequado. Também é importante consultar o obstetra para se certificar de que não existem fatores de risco fetais que possam afetar a decisão de usar farmacoterapias específicas.

Em conclusão, este caso clínico retrata uma doente com EN diagnosticado no primeiro trimestre de gravidez e chama a atenção para o desafio constante que é para o médico de família o diagnóstico diferencial de alterações cutâneas, no contexto da gravidez. Neste sentido, é importante ter em mente as patologias cutâneas mais frequentes nos cuidados de saúde primários; porém, deve ser sempre mantido um grau de suspeição elevado quanto à possibilidade de aparecimento de alterações cutâneas menos comuns. Isto torna-se particularmente relevante no âmbito da saúde materna, dada a importância de evitar submeter as gestantes e o embrião/feto a meios complementares de diagnóstico e terapêuticas farmacológicas desnecessárias.

\section{REFERÊNCIAS BIBLIOGRÁFICAS}

1. Tunzi M, Gray GR. Common skin conditions during pregnancy. Am Fam Physician. 2007;75(2):211-8.

2. Fernandes $L B$, Mendonça $C R$, Amaral WN. Alterações dermatológicas na gravidez: revisão da literatura [Skin changes in pregnancy: literature review]. Femina. 2014;42(2):101-8. Portuguese

3. Shornick J. Pregnancy dermatoses. In: Bolognia JL, Jorizzo JL, Rapini RP, editors. Dermatology. 2nd ed. London: Mosby Elsevier; 2008. p. 142135. ISBN 9781416029991

4. Tavares E, Fernandes E, Martins C. Dermatoses específicas da gravidez [Specific skin diseases associated with pregnancy].Acta Obstet Ginecol Port. 2011;5(3):122-31. Portuguese

5. Jhaveri K, Halperin P, Shin SJ, Vahdat L. Erythema nodosum secondary to aromatase inhibitor use in breast cancer patients: case reports and review of the literature. Breast Cancer Res Treat. 2007;106(3):315-8.

6. Acosta KA, Haver MC, Kelly B. Etiology and therapeutic management of erythema nodosum during pregnancy: an update. Am J Clin Dermatol. 2013;14(3):215-22.

7. Bartelsmeyer JA, Petrie RH. Erythema nodosum, estrogens, and pregnancy. Clin Obstet Gynecol. 1990;33(4):777-81.

8. Schwartz RA, Nervi SJ. Erythema nodosum: a sign of systemic disease. Am Fam Physician. 2007;75(5):695-700.

\section{CONFLITO DE INTERESSES}

Os autores declaram não ter quaisquer conflitos de interesse.

\author{
ENDEREÇO PARA CORRESPONDÊNCIA \\ Carina Manuela Machado Pereira \\ E-mail: mmp.carina@gmail.com \\ https://orcid.org/0000-0003-3572-0027
}

Recebido em 13-07-2017

Aceite para publicação em 03-10-2018 


\section{ABSTRACT}

\section{ERYTHEMA NODOSUM: A CASE REPORT}

Introduction: Pregnancy, being a period of deep physiological changes, frequently leads to skin changes with a challenging differential diagnosis, mainly when concerning less common pathologies. Throughout pregnancy, numerous skin changes may occur, physiological, pregnancy-specific, and those that occur as result of the aggravation or improvement of pre-existing dermatosis.

Clinical case description: 26-year-old woman, pregnant, G3P2, surveilled at a Family Health Unit, with a personal history of asthma since childhood, currently controlled, with no relevant family history. At the 11th week of gestation, the patient had a walk-in visit due to the appearance of an erythematous lesion in the lower limb, with progressive growth, painful to the touch, non-itchy, associated with redness and edema for one week. As the hypothesis of thrombophlebitis was initially considered, the patient was referred to the $A \& E$, where the diagnosis of erythema nodosum of pregnancy was made.

Comment: The clinical case described by the author points out the skin changes that frequently occur in the context of maternal health consultations in primary health care, drawing attention to a less common alteration, the erythema nodosum. This pathology is a skin change with several possible etiological factors; its recognition guides the physician, reassures the pregnant woman and allows the implementation of an adequate management, given the benign and self-limited nature of this clinical presentation.

Keywords: Pregnancy; Erythema nodosum. 